International Mathematical Forum, 2, 2007, no. 31, 1525 - 1536

\title{
Some New Results About Shifted Hazard and Shifted Likelihood Ratio Orders
}

\author{
F. Aboukalam ${ }^{12}$ and M. Kayid \\ King Saud University, College of Science \\ Dept. of Statistics and Operation Research \\ P.O. Box 2455, Riyadh 11451, Saudi Arabia
}

\begin{abstract}
Shifted stochastic orders are a useful tool for establishing interesting inequalities. Two such stochastic orders are the up hazard rate order and the up likelihood ratio order. In this article, we give some results on the preservation of the above orderings between the components under the formation of coherent systems with different structures. Both the cases when components either identically distributed or not necessary identically distributed are discussed. Finally, we provide some applications to Poisson and non-homogeneous Poisson shock models.
\end{abstract}

Keywords: Hazard rate order, likelihood ratio order, coherent systems, Poisson shock models

\section{Introduction}

Stochastic comparisons between probability distributions play a fundamental role in probability, statistics and some related areas, such as reliability theory, survival analysis, economics and actuarial science. As a result, several more refined orders have been comprehensively discussed in the literature. The most widely used among these stochastic order relations are likelihood ratio ordering, hazard rate ordering and reversed hazard rate ordering. For a recent

\footnotetext{
${ }^{1}$ This work is support by College of Science-Research Center Project No (Stat/2007/09).

${ }^{2}$ The old name of F. Aboukalam was M. A. Fayyez Aboukalam.
} 
literature on new stochastic orders as well as others we refer the readers to Kochar, Li and Shaked (2002), Kayid and Ahmad (2004), Ahmad, Kayid and Li (2005), Ahmad, Kayid and Pellerey (2005) and Ahmad and Kayid (2005). Much of the earlier literature is cited in those papers where definitions, interrelations and discussions of many orders are presented.

Some shifted stochastic orders have been introduced and studied in Nakai (1995) and Brown and Shanthikumar (1998). They have also been touched upon in Belzunce, Lillo, Ruiz, and Shaked (2001). Recently, Lillo et al. (2001) have studied in detail four shifted stochastic orders, namely the up likelihood ratio order, the down likelihood ratio order, the up hazard rate order, and the down hazard rate order, that have been obtained starting from the well-known likelihood ratio order and hazard rate order. Along a similar line, Di Crescenzo and Longobardi (2001) proposed the up reversed hazard rate order, that is a shifted version of the reversed hazard rate order.

In this paper, we further investigate more properties of the up hazard rate order and the up likelihood ratio order, whose definitions are recalled in the next section. Section 2, contains definitions, notations and basic properties used throughout the paper. In Section 3, we give some results on the preservation of the above orderings between the components under the formation of coherent systems with different structures. Both the cases when components either identically distributed or not necessary identically distributed are discussed. Some applications to Poisson and non-homogeneous Poisson shock models are given in Section 4.

Throughout the paper we will use the term increasing in place of nondecreasing, and decreasing in place of non-increasing. Moreover, given any random variable $X$, with distribution $F$, we denote with $\bar{F}(t)=1-F(t)$ its survival function, and we always assume that $\bar{F}(0)=1$.

\section{Preliminaries}

In this section we present definitions, notations and basic properties used throughout the paper. Let $X$ and $Y$ be continuous random variables having respectively distribution function $F_{X}$ and $F_{Y}$, survival function $\bar{F}_{X}$ and $\bar{F}_{Y}$, and support $\left(l_{X}, u_{X}\right)$ and $\left(l_{Y}, u_{Y}\right)$, with $-\infty \leq l_{X}<u_{X} \leq \infty$ and $-\infty \leq$ 
$l_{Y}<u_{Y} \leq \infty$. When $X$ and $Y$ will be assumed absolutely continuous, their probability density functions will be denoted respectively by $f_{X}$ and $f_{Y}$. Below, we preliminary recall the definitions of two well-known stochastic ordering (see, for instance, Shaked and Shanthikumar (1994)).

\section{Definition 2.1.}

A random variable $X$ is smaller than a random variable $Y$ in the

(i) hazard rate order (denoted by $X \leq_{h r} Y$ ) if $\bar{F}_{X}(t) / \bar{F}_{Y}(t)$ is decreasing in $t \in\left(-\infty, \max \left\{u_{X}, u_{Y}\right\}\right)$

(ii) likelihood ratio order (denoted by $X \leq_{l r} Y$ ) if $f_{X}(t) / f_{Y}(t)$ is decreasing in $t \in\left(l_{X}, u_{Y}\right)$,provided that $X$ and $Y$ are absolutely continuous.

Recently, Lillo et al. (2001) have analyzed four stochastic orders obtained as shifted versions of $\leq_{h r}$-order and $\leq_{l r}$-order. Let us recall two of such orders which will considered in this paper.

\section{Definition 2.2.}

A random variable $X$ is smaller than a random variable $Y$ in the (i) up hazard rate order (denoted by $X \leq_{h r \uparrow} Y$ ) if

$$
X-x \leq_{h r} Y \text { for each } x \geq 0
$$

(ii) up likelihood ratio order (denoted by $X \leq_{l r \uparrow} Y$ ) if

$$
X-x \leq_{l r} Y \quad \text { for each } x \geq 0 \text {. }
$$

Thus, we observe that $X \leq_{h r \uparrow} Y$ if, and only if, for each $x \geq 0$ we have

$$
\frac{\bar{F}_{X}(t+x)}{\bar{F}_{Y}(t)} \text { is decreasing in } t \in\left(-\infty, u_{Y}\right) \text {, }
$$

and $X \leq_{l r \uparrow} Y$ if, and only if, for each $x \geq 0$ we have

$$
\frac{f_{X}(t+x)}{f_{Y}(t)} \text { is decreasing in } t \in\left(l_{X}-x, u_{X}-x\right) \cup\left(l_{Y}, u_{Y}\right) \text {, }
$$

provided that $X$ and $Y$ are absolutely continuous.

In the next section, we shall make use of the following result, that is an immediate consequence of Theorem 6.18 of Lillo et al. (2001). 


\section{Corollary 2.1.}

Let $X$ and $Y$ be continuous random variables; if $X \leq_{h r \uparrow} Y$, then

$$
\bar{F}_{X}(t+z) \geq \bar{F}_{Y}(t) \text { for all } t \in\left(-\infty, u_{Y}\right) \text { and for all } z \geq 0 .
$$

Note that if $X$ and $Y$ are absolutely continuous random variables, Theorem 6.21 of Lillo et al. (2001) yields the following characterization:

$$
X \leq_{h r \uparrow} Y \Longleftrightarrow r_{X}(w) \leq r_{Y}(v), \quad \text { whenever } l_{Y}<v \leq w<u_{X},
$$

where $r_{X}(x)=f(x) / \bar{F}(x)$, is known as the hazard rate of $X$.

The relations holding among the two shifted orders, considered in this paper, and the corresponding usual ones in the case of absolutely continuous random variables $X$ and $Y$ as follow:

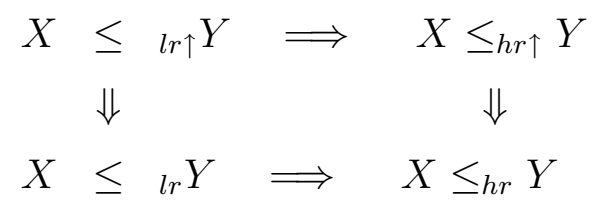

\section{Comparison of coherent systems}

Consider a system consisting of $n$ components and a structure function $\phi$. Let $h\left(p_{1}, p_{2}, \ldots, p_{n}\right)$ denote the system reliability function of structure $\phi$ (see Barlow and Proschan (1981) for definition). Define $X_{i}(t)$, the indicator random variable for the $i$ th component as

$$
X_{i}(t)= \begin{cases}1 & \text { if the component is functioning at time } t \\ 0 & \text { otherwise. }\end{cases}
$$

Let us assume that the lifetime $T_{i}$ of the $i$ th component is an absolutely continuous random variables with distribution functions $F_{i}(t)$, survival functions $\bar{F}_{i}(t)$ and density $f_{i}(t), i=1,2, \ldots, n$. If $\tau$ denotes the life of the system, then

$$
P(\tau>t)=P\left[\tau\left(T_{1}, T_{2}, \ldots, T_{n}\right)>t\right]
$$




$$
\begin{aligned}
& =P(\phi(\mathbf{X}(t))=1) \\
& =h\left(\bar{F}_{1}(t), \bar{F}_{2}(t), \ldots, \bar{F}_{n}(t)\right) \\
& =h(\overline{\mathbf{F}}(t)) .
\end{aligned}
$$

We assume that the components are independent and the system $(C, \phi)$ is coherent system (a system whose structure function $\phi$ is increasing in each argument and where each component is relevant). If the components are identically distributed, we write $h(\bar{F}(t))$ in place of $h(\bar{F}(t))$.

\subsection{Systems with i.i.d. components}

Let us consider two coherent systems $C_{1}$ and $C_{2}$ each consisting of $n$ i.i.d. components. Suppose the lifetime of the components from $C_{1}$ has distribution function $F$ and that from system $C_{2}$ has distribution function $G$. In the following result, the preservation of $\leq_{l r \uparrow}$-order is established for a coherent system with identically distributed components. This is an analogous of Theorem 2.4 of Nanda et al. (1998), where the same result is presented in the case of $\leq_{l r^{-}}$ order.

\section{Theorem 3.1.}

Let $h(p)$ be the reliability function of coherent system of $n$ independent and identical components having first and second derivatives $h^{\prime}(p)$ and $h^{\prime \prime}(p)$ respectively. If $p h^{\prime \prime}(p) / h^{\prime}(p)$ is decreasing in $p$, then

$$
h(\bar{G}) \leq_{l r \uparrow} h(\bar{F}) \quad \text { whenever } \bar{G} \leq_{l r \uparrow} \bar{F} .
$$

\section{Proof.}

First note that $h(\bar{G}) \leq_{l r \uparrow} h(\bar{F})$ if and only if

$$
\frac{f(t+z) h^{\prime}(\bar{F}(t+z)}{g(t) h^{\prime}(\bar{G}(t))} \text { is increasing in } t>0 \text {. }
$$

Assuming that $f(t+z)$ and $g(t)$ are differentiable, (3.2) is equivalent to

$$
\begin{aligned}
& {\left[f^{\prime}(t+z) g(t)-g^{\prime}(t) f(t+z)\right] h^{\prime}\left((\bar{F}(t+z)) h^{\prime}(\bar{G}(t))\right.} \\
& +f(t+z) g(t) \cdot\left[g(t) h^{\prime \prime}(\bar{G}(t)) h^{\prime}(\bar{F}(t+z))-f(t+z) h^{\prime \prime}(\bar{F}(t+z)) h^{\prime}(\bar{G}(t))\right] \geq 0,
\end{aligned}
$$


or equivalently

$$
\begin{aligned}
& {\left[f^{\prime}(t+z) g(t)-g^{\prime}(t) f(t+z)\right]} \\
& +f(t+z) g(t) \cdot\left[\frac{g(t)}{\bar{G}(t)} \cdot \frac{\bar{G}(t) h^{\prime \prime}(\bar{G}(t))}{h^{\prime}(\bar{G}(t))}\right. \\
& \left.\quad-\frac{f(t+z)}{\bar{F}(t+z)} \cdot \frac{\bar{F}(t+z) h^{\prime \prime}(\bar{F}(t+z))}{h^{\prime}((\bar{F}(t+z))}\right] \geq 0,
\end{aligned}
$$

which is non-negative because the both terms are non-negative by assumption, Corollary 2.1. and the fact that $\leq_{l r \uparrow}$-order is stronger than the $\leq_{h r \uparrow}$ order.

We aim to compare the above system with a system with identical structure, the random lifetimes of its components being now identical distributed to an absolutely continuous random lifetime $Y$. Thus, $h(\mathbf{Y})$ will denote the random lifetime of this system and $r_{h(\mathbf{X})}(t)$ its hazard rate function.

\subsection{Systems with nonidentical components}

Consider a system of $n$ independent and not necessarily identical components in which the $i$ th component has survival function $\bar{F}_{i}(t)=1-F_{i}(t)$, $i=1,2, \ldots, n$. Let $h(\mathbf{p})=h\left(p_{1}, p_{2}, \ldots, p_{n}\right)$ be the system reliability function. In the following theorem we compare the random lifetimes of two systems accord-

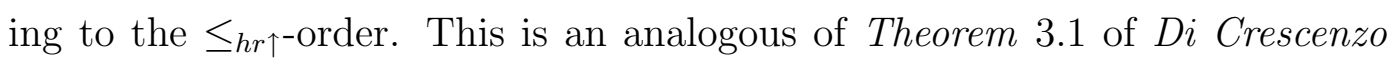
and Longobardi (2001), where the same result is present in the case of up reversed hazard order.

\section{Theorem 3.1.}

If

$$
\sum_{i=1}^{n} \frac{p_{i} \partial h / \partial p_{i}}{h(\mathbf{p})} \text { is decreasing in } p_{i} \text { for all } i=1,2, \ldots, n,
$$

then $h(\mathbf{X}) \leq_{h r \uparrow} h(Y)$ whenever $X_{i} \leq_{h r \uparrow} Y, \quad i=1,2, \ldots, n$.

\section{Proof.}

Recalling the expression of the hazard rate function of a coherent system (see (4.2) of Belzunce, Franco and Ruiz (2001), for instance), for $z>0$ and $t \geq 0$ we have:

$$
r_{h(\mathbf{X})}(z+t)=\left.\sum_{i=1}^{n} r_{X_{i}}(z+t) \bar{F}_{X_{i}}(z+t) \frac{\partial h / \partial p_{i}}{h(\mathbf{p})}\right|_{p_{i}=\bar{F}_{X_{i}}(z+t)} .
$$


Hence, due to relation (2.4), hypothesis $X_{i} \leq_{h r \uparrow} Y$ gives

$$
r_{h(\mathbf{X})}(z+t) \geq\left. r_{Y}(z) \sum_{i=1}^{n} \bar{F}_{X_{i}}(z+t) \frac{\partial h / \partial p_{i}}{h(\mathbf{p})}\right|_{p_{i}=\bar{F}_{X_{i}}(z+t)} .
$$

Using (3.1) together (2.3), implies that

$$
r_{h(\mathbf{X})}(z+t) \geq\left. r_{Y}(z) \sum_{i=1}^{n} \bar{F}_{Y}(z) \frac{\partial h / \partial p_{i}}{h(\mathbf{p})}\right|_{p_{i}=\bar{F}_{X_{i}}(z+t)} \equiv r_{h(\mathbf{Y})}(z) .
$$

This concludes the proof by virtue of (2.4).

As noted in Di Crescenzo and Longobardi (2001), the assumption (3.1) is satisfied by several coherent systems, including the $k$-out-of- $n$ one (see also Nanda et al. (1998) for more details).

\section{Poisson shock models}

The class of shock models arises in reliability theory when a system or an item is subjected to random shocks over time. The system is assumed to have an ability to withstand a random number of these shocks, and it is commonly assumed that the number of shocks and the interarival times of shocks are independent. If we denote by $N$ the number of shocks survived by the system and by $X_{j}$ the random interarival time between the $(j-1)$-th and $j$-th shocks, then the lifetime $T$ of the system is given by $T=\sum_{j=1}^{N} X_{j}$. Therefore shock models are particular cases of random sums. In particular, if the interarrivals are assumed to be independent and exponentially distributed (with common parameter $\lambda$ ), then the distribution function of $T$ can be written as

$$
H(t)=\sum_{k=0}^{\infty} \frac{e^{-\lambda t}(\lambda t)^{k}}{k !} P_{k}, \quad t \geq 0
$$

where $P_{k}=P[N \leq k]$ for all $k \in N\left(\right.$ and $\left.P_{0}=1\right)$.

Shock models of this kind, called Poisson shock models, have been studied extensively in the literature. Some recent references are Alzaid et al. (1991), Pellerey (1994), Fagiuoli and Pellerey (1994), Shaked and Wong (1995) and Belzunce et al. (1999), among others.

Suppose that a device is subjected to shocks occurring randomly as events in a Poisson process with constant $\lambda$. Supposes further that the device has 
probability $\bar{P}_{k}$ of surviving the first $k$ shock, where $1=\bar{P}_{0} \geq \bar{P}_{1} \geq \ldots$. The survival function of the device is given by

$$
\bar{F}(t)=\sum_{k=0}^{\infty} \frac{e^{-\lambda t}(\lambda t)^{k}}{k !} \bar{P}_{k} .
$$

Let $p_{k+1}=\bar{P}_{k}-\bar{P}_{k+1}, k=0,1,2, \ldots$, and let $f(t)$ be the probability function corresponding to survival function $\bar{F}(t)$, such that

$$
f(t)=\sum_{k=0}^{\infty} \frac{e^{-\lambda t}(\lambda t)^{k}}{k !} \lambda p_{k+1} .
$$

Consider another device which is also subjected to shocks occurring randomly as events in a Poisson process with the same constant intensity and the device has probability of surviving first $k$ shock, where $1=\bar{Q}_{0} \geq \bar{Q}_{1} \geq \ldots$. The survival function of this device is given by

$$
\bar{G}(t)=\sum_{k=0}^{\infty} \frac{e^{-\lambda t}(\lambda t)^{k}}{k !} \bar{Q}_{k} .
$$

Let $q_{k+1}=\bar{Q}_{k}-\bar{Q}_{k+1}, k=0,1,2, \ldots$, and let $g(t)$ be the probability density function corresponding to the survival function given by

$$
g(t)=\sum_{k=0}^{\infty} \frac{e^{-\lambda t}(\lambda t)^{k}}{k !} \lambda q_{k+1} .
$$

Singh and Jain (1989) and Kebir (1994) have shown that some partial orderings between the two shock survival probabilities $\bar{P}_{k}$ and $\bar{Q}_{k}$ are preserved by the corresponding survival functions $\bar{F}(t)$ and $\bar{G}(t)$ of the devices.

Along the same line, in the following results we give conditions on $\bar{P}_{k}$ and $\bar{Q}_{k}$ such that the lifetimes $X$ and $Y$ are ordered according to $\leq_{h r \uparrow}$-order. The proof is based on classical results of total positivity (see Karlin (1968)).

\section{Theorem 4.1.}

Let $X$ and $Y$ have distribution functions defined as in (4.1) and (4.3), respectively. If the ratio

$$
\frac{\bar{P}_{k+j}}{\bar{Q}_{k}} \text { is decreasing in } k \text { for all } j \geq 0,
$$

then $X \leq_{h r \uparrow} Y$. 


\section{Proof.}

From (4.1) and (4.3), for any real $c$, we have

$$
\bar{F}(t+s)-c \bar{G}(t)=\sum_{j=0}^{\infty} \frac{e^{-\lambda s}(\lambda s)^{j}}{j !} \sum_{k=0}^{\infty} \frac{e^{-\lambda t}(\lambda t)^{k}}{k !}\left(\bar{P}_{k+j}-c \bar{Q}_{j}\right) .
$$

Assumption (4.5) implies that $\bar{P}_{k+j}-c \bar{Q}_{j}$ has at most one change of sign; if one change occurs, it occurs fro + to - . Making use of the variation diminishing property of $e^{-\lambda t}(\lambda t)^{k} / k$ !, due to (4.6) the same statement holds for $\bar{F}(t+s)-c \bar{G}(t)$ as a function of $t$. This implies that $\bar{F}(t+s) / \bar{G}(t)$ is decreasing in $t \geq 0$. The proof then follows from (2.1).

\section{Theorem 4.2.}

Let $X$ and $Y$ have distribution functions defined as in (4.1) and (4.2), respectively. If the ratio

$$
\frac{p_{k+j+1}}{q_{k+1}} \text { is decreasing in } k \text { for all } j \geq 0,
$$

then $X \leq_{l r \uparrow} Y$.

\section{Proof.}

From (4.2) and (4.4), for any real $c$, we have

$$
f(t+s)-c g(t)=\sum_{j=0}^{\infty} \frac{e^{-\lambda s}(\lambda s)^{j}}{j !} \sum_{k=0}^{\infty} \frac{e^{-\lambda t}(\lambda t)^{k}}{k !}\left(p_{k+j+1}-c q_{j+1}\right) .
$$

By using similar arguments as in Theorem 4.1, it follows that $f(t+s) / g(t)$ is decreasing in $t \geq 0$. The proof then follows from (2.2).

We consider now non-homogeneous Poisson shock models. Let $\lambda(t)$ be the rate of the non-homogeneous Poisson counting process. Then the distribution of the random lifetime $T$ of the device is

$$
H(t)=\sum_{k=0}^{\infty} P_{k} \frac{\Lambda^{k}(t)}{k !} e^{-\Lambda(t)}
$$

where, $\Lambda(t)=\int_{0}^{t} \lambda(u) d u$ is the cumulative rate of occurrence (A-Hameed and Proschan, 1973). The reliability functions of the devices are given by

$$
\bar{F}(t)=\sum_{k=0}^{\infty} \frac{e^{-\Lambda t} \Lambda^{k}(t)}{k !} \bar{P}_{k}
$$


and

$$
\bar{G}(t)=\sum_{k=0}^{\infty} \frac{e^{-\Lambda t} \Lambda^{k}(t)}{k !} \bar{Q}_{k} .
$$

To state the closure of the up hazard rate order under non-homogeneous Poisson shock models we need the following preliminary result.

\section{Lemma 4.1.}

Assume $X \leq_{h r \uparrow} Y$, if $g(x)$ is increasing and sub-additive, then $g(X) \leq_{h r \uparrow}$ $g(Y)$.

\section{Proof.}

First not that $X \leq_{h r \uparrow} Y$ if and only if $X-x \leq_{h r} X$ for all $x>0$. By Theorem 1.B.2 (Shaked and Shathikumar, 1994) for every increasing and subadditive function $g$, we have

$$
g(X-x) \leq g(Y)
$$

Because of subadditivity,

$$
g(X-x)+g(x) \geq_{h r} g(X) .
$$

Thus,

$$
g(X)-g(x) \leq_{h r} g(Y) .
$$

Now, from the arbitrariness of $x$ it follows that

$$
g(X) \leq_{h r \uparrow} g(Y) .
$$

According to Theorem 4.1, Lemma 4.1, Theorem 4.3 below is immediate.

\section{Theorem 4.3.}

Let $X$ and $Y$ have distribution functions defined as in (4.7) and (4.8), respectively. If the ratio

$$
\bar{P}_{k+j} / \bar{Q}_{k} \text { decreases in } k \text { for al } l j \geq 0,
$$

then

$$
X \leq_{h r \uparrow} Y .
$$

\section{REFERENCES}

Ahmad, I. A. and Kayid, M. (2005). Characterizations of the RHR and MIT 
orderings and the DRHR and IMIT classes of life distributions. Probability in the Engineering and Informational Sciences, 19, 447-461.

Ahmad, I. A., Kayid, M. and Li, X. (2005). The NBUT class of life distributions. IEEE Transaction on Reliability, 396-401.

Ahmad, I. A., Kayid, M. and Pellerey, F. (2005). Further results involving the MIT order and the IMIT class. Probability in the Engineering and Informational Sciences, 19, 377-395.

A-Hameed, M. S. and Proschan, F. (1973). Nonstationary shock models. Stochastic Processes Applications, 1, 33-404.

Alzaid, A., Kim, J. S. and Proschan, F. (1991). Laplace ordering and its applications. Journal of Applied Probability, 28, 116-130.

Barlow, R. E. and Proschan, F. (1981). Statistical Theory of Reliability and Life Testing. To Begin with. Silver Spring, M D.

Belzunce, F., Franco, M. and Ruiz, J. M. (2001). On partial orderings between coherent systems with different structures. Probability in the Engineering and Informational Sciences, 15, 273-293.

Belzunce, F., Lillo, R., Ruiz, J. M. and Shaked, M. (2001). Stochastic comparisons of nonhomogeneous processes. Probability in the Engineering and Informational Sciences, 15, 199-224.

Belzunce, F., Ortega, E. and Ruiz, J. M. (1999). The Laplace order and ordering of residual lives. Statistics \& Probability Letters, 42, 145-156.

Brown, M. and Shanthikumar, J. G. (1998). Comparing the variability of random variables and point processes. Probability in the Engineering and Informational Sciences, 12, 425-444.

Di Crescenzo, A. and Longobardi, M. (2001). The up reversed hazard rate stochastic order. Scientiae Mathematicae Japonica Online, 4, 969-976.

Fagiuoli, E. and Pellerey, F. (1994). Mean residual life and increasing convex comparison of shock models. Statistics \& Probability Letters, 20, 337-345.

Kayid, M. and Ahmad, I. A. (2004). On the mean inactivity time ordering with reliability applications. Probability in the Engineering and Informational Sciences, 18, 395-409. 
Karlin, S. (1968). Total Positivity. Vol I. Standford University Press, Stanford C A.

Kebir, Y. (1994). Order-preserving shock models. Probability in the Engineering and Informational Sciences, 8, 125-134.

Kochar, S. C., Li, X. and Shaked, M. (2002). The total time on test transform and the excess wealth stochastic orders of distributions. Advances in Applied Probability, 34, 826-845.

Lillo, R. E., Nanda, A. K. and Shaked, M. (2001). Some shifted stochastic orders. In: Recent Advances in Reliability Theory: Methodology, Practice, (Limnios N. and Nikulin M., eds.), pp. 85-103, Birkhauser, Boston.

Nanda, A. K., Jain, K. and Singh, H. (1998). Preservation of some partial orderings under the formation of coherent systems. Statistics \& Probability Letters, 39, 123-131.

Nakai, T. (1995). A partially observable decision problem under a shifted likelihood ratio ordering. Mathematical and Computer Modelling, 22, 237-246.

Pellerey, F. (1994). Shock models with underlying counting process. Journal of Applied Probability, 31, 156-166.

Shaked, M. and Shanthikumar, J. G. (1994). Stochastic Orders and Their Applications. Academic Press, New York.

Shaked, M. and Wong, T. (1995). Preservation of stochastic orderings under random mapping by point processes. Probability in the Engineering and Informational Sciences, 9, 563-580.

Singh, H. and Jain, K. (1989). Preservation of some partial orderings under Poisson shock models. Journal of Applied Probability, 21, 713-716.

\section{Received: May 23, 2006}

